\title{
Fusion and fission barrier heights and positions within the Generalized Liquid Drop Model
}

\author{
G. Royer, M. Guillot, J. Monard \\ Laboratoire Subatech, UMR:IN2P3/CNRS-Université-IMT, 44-Nantes, France
}

\begin{abstract}
The fusion and fission barriers have been determined with a Generalized Liquid Drop Model taking into account the proximity forces acting between surfaces in regard, the charge and mass asymmetry, the shell and pairing effects and quasimolecular shapes. The heights and positions of these barriers have been compared with the empirical results deduced from the experimental data. There is an overall agreement for the fusion barrier heights for most of the reactions. These fusion barrier heights may also be calculated from a proposed analytic formula or other formulas. The empirical fission barrier heights lie always between the values given by the GLDM and neglecting the microscopic effects in the fragments and the values determined in including the deformation and shell and pairing effects of the fragments.
\end{abstract}

Keywords: Fission, Fusion, Potential barriers, Generalized Liquid Drop Model.

\section{Introduction}

To predict and determine the cross sections of fusion reactions it is, firstly, needful to extract the fusion barrier height and radius from the fusion barrier leading the two incoming nuclei till the compound nucleus. To calculate the fission probability and half-lives of nuclei it is necessary to know the potential barrier from the ground state till two well separated fragments.

The Generalized Liquid Drop Model, previously used, has allowed to reproduce some of the fusion [1-3], fission [4,5], cluster [6,7] and $\alpha$ radioactivity $[8,9]$ data. Assuming volume conservation, this GLDM takes into account the proximity forces acting between surfaces in regard, the charge and mass asymmetry and the shell and pairing effects. Its complete description is displayed, particularly,

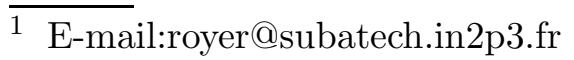


in $[1,5,10,11]$.

The quasimolecular shape sequence (elliptic lemniscatoids) previously used to describe the path leading from two spherical nuclei to one sphere or vice-versa has been retained once again to investigate these fusion and fission reactions [10]. It allows to describe smoothly the formation of a deep neck while keeping almost spherical ends and, then, a smooth transition between the two-body and one-body shapes or vice-versa. For the fission study, the path through ellipsoidal deformations of the fragments has also been investigated.

The purpose of this work is to provide the fusion and fission barrier heights and positions extracted from the fusion and fission valleys determined by the GLDM and the quasimolecular one-body shapes and the two separated spherical or ellipsoidal shapes and to compare with the experimental data as well as values obtained from analytic formulas for the fusion data.

\section{Fusion barrier}

The fusion reactions of two ions are the principal way to create exotic nuclei far from the $\beta$ stability valley $[12,13]$ and isotopes of superheavy elements [14-17]. Recently, the barriers of the fusion reactions leading possibly to the synthesis of some superheavy nuclei have been determined within the GLDM [3].

Here, the macroscopic fusion barriers for 3 reactions are displayed in Fig. 1. The incoming nuclei are supposed to be spherical and the shell effects are disregarded. The saddle-point corresponds always to two separated incoming nuclei maintained in unstable equilibrium by the balance between the repulsive Coulomb forces and the attractive proximity forces. The formation of the neck occurs, after, in the descent till the ground state. For heavier systems $\left(Z_{1} Z_{2} \geq 1800_{-}^{+} 100\right)$, even macroscopically, the proximity effects lead to an inflection in the potential energy curves and to the appearance of a double-humped macroscopic fusion barriers. The inner barrier is the highest for $Z_{1} Z_{2} \geq 2300_{-}^{+} 100[1-3]$.

In Table 1 the empirical fusion barrier heights $E_{\exp }$ and radii $R_{\exp }$ deduced from the experimental data (extracted from [1,2,18-31]) are given for most of the experimentally investigated systems. The values obtained using the macroscopic part of the GLDM [1] are compared, firstly (in $5^{\text {th }}$ and $6^{\text {th }}$ columns), with these data and with the values calculated from our following formulas (in $7^{\text {th }}$ and $8^{\text {th }}$ columns) adjusted on GLDM data for 170 fusion reactions [18].

$$
E=-19.4+\frac{2.1388 Z_{1} Z_{2}+59.427\left(A_{1}^{1 / 3}+A_{2}^{1 / 3}\right)-27.07 \ln \left(\frac{Z_{1} Z_{2}}{A_{1}^{1 / 3}+A_{2}^{1 / 3}}\right)}{\left(A_{1}^{1 / 3}+A_{2}^{1 / 3}\right)\left(2.97-0.12 \ln \left(Z_{1} Z_{2}\right)\right)} .
$$




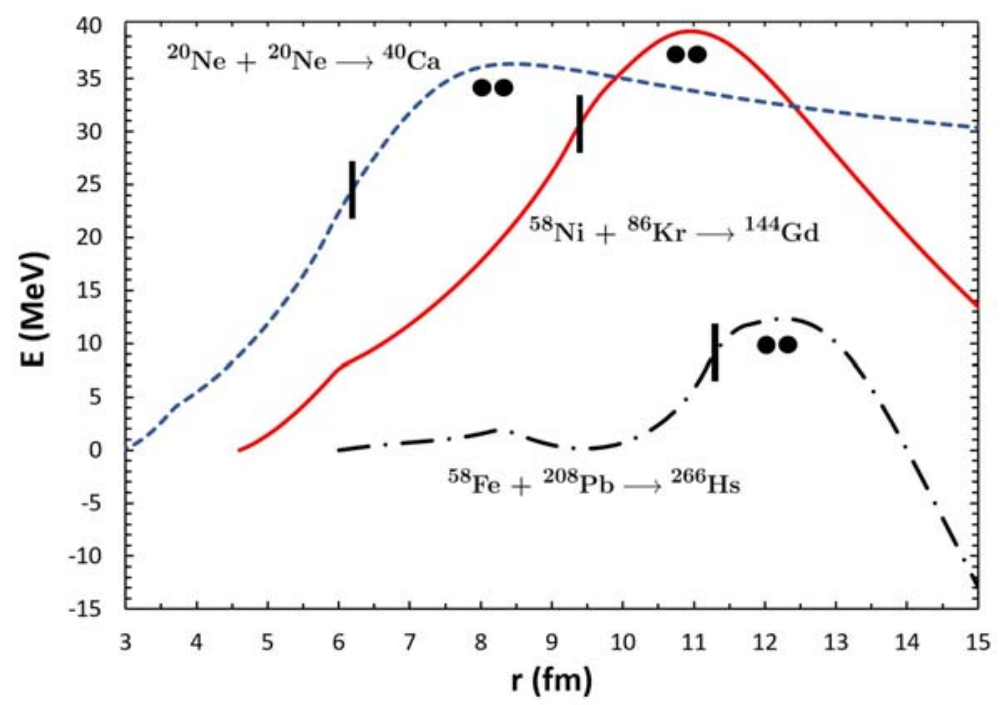

Fig. 1. Potential barriers governing the ${ }^{20} \mathrm{Ne}+{ }^{20} \mathrm{Ne},{ }^{58} \mathrm{Ni}+{ }^{86} \mathrm{Kr}$ and ${ }^{58} \mathrm{Fe}+{ }^{208} \mathrm{~Pb}$ fusion reactions versus the distance between the mass centres. The vertical dash indicates the contact point between the two nuclei.

$$
R=\left(A_{1}^{1 / 3}+A_{2}^{1 / 3}\right)\left[1.908-0.0857 \ln \left(Z_{1} Z_{2}\right)+\frac{3.94}{Z_{1} Z_{2}}\right] .
$$

It is found in Ref. [32] that the parameterized formulas given in [33-35] seem to be more suitable for description of the experimental fusion cross sections at energies below the Coulomb barrier. So, for comparison, these formulas are recalled here.

The proposed formulas for the fusion barrier heights and positions in $[32,33]$ are

$$
\begin{aligned}
& E=1.327 \frac{Z_{1} Z_{2}}{R} \\
& R=2.386+1.099\left(<r_{1}^{2}>^{1 / 2}+<r_{2}^{2}>^{1 / 2}\right)
\end{aligned}
$$

where the root-mean-square radii $\left\langle r_{i}^{2}>^{1 / 2}\right.$ of the reacting nuclei may be evaluated from the experimental data or from a formula. Here the following expression proposed to reproduce accurately the Coulomb radius has been retained [36].

$$
<r_{i}^{2}>^{1 / 2}=0.6^{1 / 2} R_{i}=0.6^{1 / 2}\left(1.2332 A_{i}^{1 / 3}+\frac{2.8961}{A_{i}^{2 / 3}}-0.18688 A_{i}^{1 / 3} I_{i}\right)
$$

where $I=(N-Z) / A$ is the relative neutron excess.

The proposed formulas for the fusion barrier heights and positions in Ref. [34] 
are

$$
\begin{aligned}
& E=\frac{Z_{1} Z_{2} e^{2}}{R}\left(1-\frac{0.63}{R}\right) . \\
& R=1.07\left(A_{1}^{1 / 3}+A_{2}^{1 / 3}\right)+2.72 .
\end{aligned}
$$

In Ref. [35] the following formulas are given

$$
\begin{gathered}
E=-1.01+\frac{0.93 Z_{1} Z_{2}}{A_{1}^{1 / 3}+A_{2}^{1 / 3}}+0.000453\left(\frac{Z_{1} Z_{2}}{A_{1}^{1 / 3}+A_{2}^{1 / 3}}\right)^{2} . \\
R=0.88\left(A_{1}^{1 / 3}+A_{2}^{1 / 3}\right)+3.58 .
\end{gathered}
$$

The two following columns of the table ( $9^{\text {th }}$ and $10^{\text {th }}$ columns) give respectively the fusion barrier heights calculated from the analytic formulas provided in Refs. [33] and [34].

Table 1: Comparison of the experimental and theoretical (GLDM values) fusion barrier heights $\mathrm{E}$ (in $\mathrm{MeV}$ ) and positions $\mathrm{R}$ (in $\mathrm{fm}$ ). The values given by different formulas are also displayed.

\begin{tabular}{|c|c|cc|cc|cc|c|c|c|}
\hline Reaction & $Z_{1} Z_{2}$ & $E_{\text {exp }}$ & $R_{\text {exp }}$ & $E_{[1]}$ & $R_{[1]}$ & $E_{[18]}$ & $R_{[18]}$ & $E_{[33]}$ & $E_{[34]}$ & Ref. \\
\hline${ }_{4}^{9} B e+{ }_{5}^{10} B$ & 20 & 3.40 & 7.65 & 3.36 & 7.79 & 3.45 & 7.83 & 3.36 & 3.63 & {$[2]$} \\
${ }_{4}^{9} B e+{ }_{6}^{12} C$ & 24 & 4.10 & 7.65 & 4.00 & 7.87 & 4.04 & 7.86 & 3.99 & 4.28 & {$[29]$} \\
${ }_{5}^{10} B+{ }_{5}^{10} B$ & 25 & 4.41 & 7.34 & 4.22 & 7.75 & 4.21 & 7.96 & 4.16 & 4.49 & {$[29]$} \\
${ }_{5}^{10} B+{ }_{5}^{11} B$ & 25 & 4.36 & 7.46 & 4.17 & 7.86 & 4.18 & 7.84 & 4.16 & 4.45 & {$[2]$} \\
${ }_{5}^{11} B+{ }_{5}^{11} B$ & 25 & 4.30 & 7.58 & 4.12 & 7.98 & 4.15 & 7.71 & 4.15 & 4.41 & {$[29]$} \\
${ }_{5}^{11} B+{ }_{6}^{12} C$ & 30 & 5.20 & 7.51 & 4.96 & 7.94 & 4.93 & 7.89 & 4.94 & 5.24 & {$[29]$} \\
${ }_{5}^{10} B+{ }_{6}^{12} C$ & 30 & 5.24 & 7.45 & 5.03 & 7.81 & 4.91 & 7.77 & 4.94 & 5.29 & {$[2]$} \\
${ }_{4}^{9} B e+{ }_{8}^{16} \mathrm{O}$ & 32 & 5.40 & 7.73 & 5.25 & 8.00 & 5.24 & 7.98 & 5.22 & 5.53 & {$[29]$} \\
${ }_{5}^{10} B+{ }_{7}^{14} N$ & 35 & 5.80 & 7.89 & 5.82 & 7.88 & 5.68 & 7.83 & 5.71 & 6.08 & {$[2]$} \\
${ }_{6}^{12} \mathrm{C}+{ }_{6}^{12} \mathrm{C}$ & 36 & 6.37 & 7.34 & 5.98 & 7.90 & 5.84 & 7.83 & 5.87 & 6.24 & {$[29]$} \\
${ }_{6}^{12} \mathrm{C}+{ }_{6}^{13} \mathrm{C}$ & 36 & 5.98 & 7.89 & 5.91 & 7.98 & 5.84 & 7.94 & 5.86 & 6.19 & {$[29]$} \\
${ }_{6}^{13} \mathrm{C}+{ }_{6}^{13} \mathrm{C}$ & 36 & 6.01 & 7.83 & 5.85 & 8.08 & 5.85 & 8.04 & 5.86 & 6.14 & {$[29]$} \\
${ }_{2}^{4} \mathrm{He}+{ }_{20}^{40} \mathrm{Ca}$ & 40 & 6.80 & 7.68 & 6.31 & 8.37 & 6.45 & 8.46 & 6.01 & 6.57 & {$[29]$}
\end{tabular}




\begin{tabular}{|c|c|c|c|c|c|c|c|c|c|c|}
\hline${ }_{2}^{4} \mathrm{He}+{ }_{20}^{44} \mathrm{Ca}$ & 40 & 6.37 & 8.25 & 6.20 & 8.51 & 6.44 & 8.65 & 5.97 & 6.49 & {$[2]$} \\
\hline${ }_{5}^{10} B+{ }_{8}^{16} O$ & 40 & 6.57 & 7.99 & 6.60 & 7.96 & 6.46 & 7.90 & 6.46 & 6.85 & [29] \\
\hline${ }_{6}^{12} C+{ }_{7}^{14} N$ & 42 & 7.04 & 7.81 & 6.92 & 7.95 & 6.77 & 7.90 & 6.78 & 7.17 & [29] \\
\hline${ }_{6}^{12} C+{ }_{7}^{15} N$ & 42 & 6.79 & 8.12 & 6.85 & 8.05 & 6.76 & 8.00 & 6.77 & 7.12 & [29] \\
\hline${ }_{6}^{12} \mathrm{C}+{ }_{8}^{16} \mathrm{O}$ & 48 & 7.95 & 7.90 & 7.85 & 8.02 & 7.69 & 7.98 & 7.68 & 8.08 & [29] \\
\hline${ }_{6}^{12} \mathrm{C}+{ }_{8}^{17} \mathrm{O}$ & 48 & 7.95 & 7.90 & 7.78 & 8.11 & 7.68 & 8.06 & 7.67 & 8.03 & [29] \\
\hline${ }_{6}^{12} \mathrm{C}+{ }_{8}^{18} \mathrm{O}$ & 48 & 7.68 & 8.23 & 7.71 & 8.20 & 7.67 & 8.14 & 7.65 & 7.98 & [29] \\
\hline${ }_{7}^{14} N+{ }_{7}^{14} N$ & 49 & 8.17 & 7.85 & 8.01 & 8.03 & 7.85 & 7.98 & 7.84 & 8.24 & {$[2]$} \\
\hline${ }_{6}^{12} C+{ }_{9}^{19} F$ & 54 & 8.51 & 8.35 & 8.69 & 8.18 & 8.59 & 8.13 & 8.55 & 8.93 & [29] \\
\hline${ }_{2}^{4} \mathrm{He}+{ }_{27}^{59} \mathrm{Co}$ & 54 & 8.26 & 8.63 & 8.10 & 8.83 & 8.40 & 8.98 & 7.77 & 8.39 & {$[2]$} \\
\hline${ }_{7}^{14} N+{ }_{8}^{16} O$ & 56 & 9.05 & 8.12 & 9.08 & 8.11 & 8.92 & 8.05 & 8.87 & 9.29 & [29] \\
\hline${ }_{8}^{16} \mathrm{O}+{ }_{8}^{16} \mathrm{O}$ & 64 & 10.74 & 7.81 & 10.29 & 8.16 & 10.14 & 8.13 & 10.05 & 10.48 & [29] \\
\hline${ }_{6}^{12} C+{ }_{13}^{27} A l$ & 78 & 12.29 & 8.36 & 12.24 & 8.40 & 12.16 & 8.38 & 11.94 & 12.40 & {$[2]$} \\
\hline${ }_{6}^{12} \mathrm{C}+{ }_{14}^{28} S i$ & 84 & 14.53 & 7.53 & 13.19 & 8.38 & 13.08 & 8.39 & 12.79 & 13.29 & {$[29]$} \\
\hline${ }_{6}^{12} \mathrm{C}+{ }_{14}^{29} S i$ & 84 & 13.46 & 8.19 & 13.11 & 8.45 & 13.04 & 8.45 & 12.77 & 13.24 & {$[29]$} \\
\hline${ }_{6}^{12} C+{ }_{14}^{30} S i$ & 84 & 13.20 & 8.39 & 13.04 & 8.51 & 13.00 & 8.50 & 12.75 & 13.18 & {$[29]$} \\
\hline${ }_{8}^{16} \mathrm{O}+{ }_{12}^{24} M g$ & 96 & 15.26 & 8.28 & 15.01 & 8.42 & 14.89 & 8.42 & 14.57 & 15.05 & [29] \\
\hline${ }_{8}^{16} \mathrm{O}+{ }_{12}^{26} \mathrm{Mg}$ & 96 & 15.24 & 8.29 & 14.82 & 8.56 & 14.78 & 8.54 & 14.54 & 14.99 & {$[2]$} \\
\hline${ }_{8}^{18} \mathrm{O}+{ }_{12}^{24} \mathrm{Mg}$ & 96 & 15.26 & 8.28 & 14.76 & 8.58 & 14.75 & 8.58 & 14.52 & 14.88 & {$[29]$} \\
\hline${ }_{9}^{19} \mathrm{~F}+{ }_{11}^{23} N a$ & 99 & 15.16 & 8.62 & 15.23 & 8.59 & 15.21 & 8.57 & 14.96 & 15.33 & {$[29]$} \\
\hline${ }_{10}^{20} N e+{ }_{10}^{20} N e$ & 100 & 15.20 & - & 15.61 & 8.44 & 15.49 & 8.43 & 15.16 & 15.64 & [19] \\
\hline${ }_{8}^{16} O+{ }_{13}^{27} A l$ & 104 & 16.04 & 8.56 & 16.05 & 8.54 & 15.99 & 8.54 & 15.64 & 16.09 & {$[29]$} \\
\hline${ }_{8}^{17} \mathrm{O}+{ }_{13}^{27} \mathrm{Al}$ & 104 & 15.74 & 8.73 & 15.91 & 8.62 & 15.91 & 8.62 & 15.61 & 16.00 & {$[29]$} \\
\hline${ }_{8}^{18} O+{ }_{13}^{27} A l$ & 104 & 15.58 & 8.84 & 15.79 & 8.70 & 15.83 & 8.70 & 15.58 & 15.91 & {$[29]$} \\
\hline${ }_{6}^{12} C+{ }_{18}^{40} A r$ & 108 & 16.2 & - & 16.27 & 8.78 & 16.31 & 8.81 & 15.91 & 16.36 & {$[2]$} \\
\hline${ }_{8}^{16} \mathrm{O}+{ }_{14}^{28} S i$ & 112 & 17.00 & 8.70 & 17.29 & 8.57 & 17.19 & 8.55 & 16.75 & 17.26 & [29] \\
\hline${ }_{8}^{16} \mathrm{O}+{ }_{14}^{29} \mathrm{Si}$ & 112 & 16.3 & 9.12 & 17.19 & 8.59 & 17.13 & 8.61 & 16.72 & 17.19 & [29] \\
\hline${ }_{8}^{16} \mathrm{O}+{ }_{14}^{30} \mathrm{Si}$ & 112 & 16.1 & 9.18 & 17.09 & 8.65 & 17.07 & 8.66 & 16.69 & 17.12 & {$[29]$} \\
\hline${ }_{8}^{18} \mathrm{O}+{ }_{14}^{28} S i$ & 112 & 16.90 & 8.76 & 17.01 & 8.72 & 17.01 & 8.71 & 16.69 & 17.06 & [29] \\
\hline
\end{tabular}




\begin{tabular}{|c|c|c|c|c|c|c|c|c|c|c|}
\hline${ }_{6}^{12} \mathrm{C}+{ }_{22}^{46} \mathrm{Ti}$ & 132 & 20.7 & 9.0 & 19.70 & 8.89 & 19.69 & 8.92 & 19.06 & 19.63 & {$[2]$} \\
\hline${ }_{6}^{12} \mathrm{C}+{ }_{22}^{48} \mathrm{Ti}$ & 132 & 20.0 & 8.0 & 19.54 & 8.96 & 19.59 & 9.00 & 19.01 & 19.52 & {$[2]$} \\
\hline${ }_{6}^{12} \mathrm{C}+{ }_{22}^{50} \mathrm{Ti}$ & 132 & 19.5 & 7.8 & 19.41 & 9.02 & 19.49 & 9.08 & 18.96 & 19.42 & {$[2]$} \\
\hline${ }_{2}^{4} \mathrm{He}+{ }_{66}^{164} \mathrm{Dy}$ & 132 & 17.14 & 10.32 & 16.98 & 10.45 & 17.61 & 10.73 & 16.39 & 17.36 & {$[2]$} \\
\hline${ }_{8}^{16} O+{ }_{18}^{40} A r$ & 144 & 21.0 & - & 21.35 & 8.95 & 21.38 & 8.97 & 20.85 & 21.26 & {$[2]$} \\
\hline${ }_{12}^{24} M g+{ }_{12}^{24} M g$ & 144 & 22.30 & - & 21.88 & 8.72 & 21.79 & 8.71 & 21.15 & 21.67 & [20] \\
\hline${ }_{8}^{16} \mathrm{O}+{ }_{20}^{40} \mathrm{Ca}$ & 160 & 23.06 & 9.21 & 23.91 & 8.86 & 23.84 & 8.90 & 23.03 & 23.62 & {$[29]$} \\
\hline${ }_{8}^{18} \mathrm{O}+{ }_{20}^{44} \mathrm{Ca}$ & 160 & 22.3 & 8.2 & 23.13 & 9.20 & 23.28 & 9.21 & 22.82 & 23.09 & {$[2]$} \\
\hline${ }_{2}^{4} \mathrm{He}+{ }_{82}^{208} \mathrm{~Pb}$ & 164 & 20.52 & 10.74 & 20.23 & 10.90 & 20.91 & 11.23 & 19.57 & 20.67 & {$[29]$} \\
\hline${ }_{2}^{4} \mathrm{He}+{ }_{83}^{209} \mathrm{Bi}$ & 166 & 20.52 & 10.88 & 20.46 & 10.93 & 21.14 & 11.23 & 19.78 & 20.9 & {$[2]$} \\
\hline${ }_{12}^{26} M g+{ }_{14}^{30} S i$ & 168 & 24.80 & - & 24.61 & 9.04 & 24.70 & 9.06 & 24.17 & 24.46 & [30] \\
\hline${ }_{2}^{4} \mathrm{He}+{ }_{92}^{233} \mathrm{U}$ & 184 & 21.69 & 11.45 & 22.23 & 11.16 & 22.93 & 11.48 & 21.51 & 22.7 & {$[2]$} \\
\hline${ }_{3}^{6} L i+{ }_{62}^{144} S m$ & 186 & 24.65 & - & 24.32 & 10.27 & 24.68 & 10.46 & 23.47 & 24.47 & {$[30]$} \\
\hline${ }_{7}^{14} N+{ }_{27}^{59} C o$ & 189 & 26.13 & - & 27.11 & 9.26 & 27.16 & 9.33 & 26.3 & 26.84 & {$[21]$} \\
\hline${ }_{12}^{24} M g+{ }_{16}^{32} S$ & 192 & 28.28 & 9.00 & 28.48 & 8.94 & 28.40 & 8.96 & 27.43 & 27.98 & {$[29]$} \\
\hline${ }_{12}^{25} M g+{ }_{16}^{32} S$ & 192 & 27.60 & 9.32 & 28.29 & 9.00 & 28.27 & 9.01 & 27.39 & 27.87 & {$[2]$} \\
\hline${ }_{12}^{26} M g+{ }_{16}^{32} S$ & 192 & 27.48 & 9.36 & 28.12 & 9.06 & 28.14 & 9.07 & 27.34 & 27.75 & {$[2]$} \\
\hline${ }_{12}^{24} M g+{ }_{16}^{34} S$ & 192 & 27.38 & 9.40 & 28.19 & 9.05 & 28.18 & 9.05 & 27.35 & 27.79 & {$[2]$} \\
\hline${ }_{12}^{25} M g+{ }_{16}^{34} S$ & 192 & 27.26 & 9.45 & 28.00 & 9.11 & 28.05 & 9.11 & 27.31 & 27.67 & {$[2]$} \\
\hline${ }_{12}^{26} M g+{ }_{16}^{34} S$ & 192 & 27.11 & 9.50 & 27.84 & 9.17 & 27.93 & 9.17 & 27.26 & 27.56 & {$[2]$} \\
\hline${ }_{3}^{7} L i+{ }_{65}^{159} \mathrm{~Tb}$ & 195 & 23.81 & 11.03 & 24.69 & 10.60 & 25.21 & 10.82 & 24.32 & 25.00 & {$[29]$} \\
\hline${ }_{14}^{28} S i+{ }_{14}^{28} S i$ & 196 & 28.67 & 9.06 & 29.04 & 8.96 & 28.97 & 8.96 & 27.98 & 28.53 & {$[29]$} \\
\hline${ }_{14}^{28} S i+{ }_{14}^{30} S i$ & 196 & 28.60 & 8.10 & 28.71 & 9.07 & 28.72 & 9.07 & 27.89 & 28.31 & {$[2]$} \\
\hline${ }_{14}^{30} S i+{ }_{14}^{30} S i$ & 196 & 28.10 & 8.60 & 28.40 & 9.18 & 28.49 & 9.17 & 27.80 & 28.10 & {$[2]$} \\
\hline${ }_{10}^{20} \mathrm{Ne}+{ }_{20}^{40} \mathrm{Ca}$ & 200 & 28.41 & 9.36 & 29.44 & 9.01 & 29.37 & 9.04 & 28.32 & 28.92 & {$[29]$} \\
\hline${ }_{12}^{24} \mathrm{Mg}+{ }_{17}^{35} \mathrm{Cl}$ & 204 & 30.70 & - & 29.94 & 9.06 & 29.90 & 9.06 & 28.92 & 29.43 & {$[30]$} \\
\hline${ }_{16}^{32} S+{ }_{13}^{27} A l$ & 208 & 30.32 & 9.10 & 30.47 & 9.07 & 30.44 & 9.07 & 29.46 & 29.94 & {$[29]$} \\
\hline${ }_{13}^{27} A l+{ }_{17}^{35} C l$ & 221 & 32.0 & 9.15 & 32.03 & 9.14 & 32.05 & 9.18 & 31.06 & 31.49 & {$[29]$} \\
\hline${ }_{8}^{16} O+{ }_{28}^{58} N i$ & 224 & 31.67 & - & 32.06 & 9.27 & 32.04 & 9.34 & 30.88 & 31.52 & {$[30$} \\
\hline
\end{tabular}




\begin{tabular}{|c|c|c|c|c|c|c|c|c|c|c|}
\hline${ }_{8}^{16} \mathrm{O}+{ }_{29}^{63} \mathrm{Cu}$ & 232 & 33.3 & 9.26 & 32.77 & 9.41 & 32.81 & 9.48 & 31.73 & 32.29 & {$[29]$} \\
\hline${ }_{6}^{12} \mathrm{C}+{ }_{46}^{110} \mathrm{Pd}$ & 276 & 35.1 & - & 36.59 & 10.11 & 36.72 & 10.20 & 35.65 & 36.24 & {$[2]$} \\
\hline${ }_{6}^{12} C+{ }_{50}^{124} S n$ & 300 & 37.4 & - & 39.01 & 10.30 & 39.15 & 10.42 & 38.13 & 38.66 & {$[2]$} \\
\hline${ }_{6}^{12} \mathrm{C}+{ }_{52}^{130} \mathrm{Te}$ & 312 & 38.6 & - & 40.26 & 10.39 & 40.40 & 10.51 & 39.38 & 39.90 & {$[2]$} \\
\hline${ }_{16}^{32} S+{ }_{20}^{40} C a$ & 320 & 43.9 & 9.72 & 45.39 & 9.37 & 45.28 & 9.40 & 43.49 & 44.10 & [29] \\
\hline${ }_{8}^{16} \mathrm{O}+{ }_{46}^{110} \mathrm{Pd}$ & 368 & 46.2 & - & 48.05 & 10.26 & 48.10 & 10.33 & 46.81 & 47.26 & {$[2]$} \\
\hline${ }_{6}^{12} C+{ }_{62}^{152} S m$ & 372 & 46.39 & 10.77 & 46.88 & 10.65 & 46.98 & 10.76 & 45.74 & 46.38 & [29] \\
\hline${ }_{17}^{35} \mathrm{Cl}+{ }_{22}^{48} \mathrm{Ti}$ & 374 & 52.3 & 9.51 & 51.23 & 9.75 & 51.28 & 9.74 & 49.68 & 49.96 & [29] \\
\hline${ }_{14}^{28} S i+{ }_{28}^{58} N i$ & 392 & 53.80 & 8.10 & 53.94 & 9.69 & 53.83 & 9.71 & 51.77 & 52.35 & [31] \\
\hline${ }_{14}^{28} S i+{ }_{28}^{62} N i$ & 392 & 52.89 & 7.70 & 53.28 & 9.83 & 53.27 & 9.84 & 51.54 & 51.90 & [31] \\
\hline${ }_{14}^{28} S i+{ }_{28}^{64} N i$ & 392 & 52.40 & 7.60 & 52.99 & 9.89 & 53.00 & 9.90 & 51.43 & 51.69 & [31] \\
\hline${ }_{14}^{30} S i+{ }_{28}^{62} N i$ & 392 & 52.20 & 9.70 & 52.72 & 9.93 & 52.82 & 9.94 & 51.39 & 51.55 & [31] \\
\hline${ }_{14}^{30} S i+{ }_{28}^{64} N i$ & 392 & 51.20 & 9.60 & 52.44 & 9.99 & 52.56 & 9.99 & 51.28 & 51.34 & [31] \\
\hline${ }_{8}^{16} \mathrm{O}+{ }_{50}^{116} S n$ & 400 & 50.94 & - & 51.90 & 10.31 & 51.91 & 10.39 & 50.38 & 50.95 & [30] \\
\hline${ }_{8}^{18} O+{ }_{50}^{124} S n$ & 400 & 49.30 & - & 50.51 & 10.61 & 50.72 & 10.68 & 49.93 & 49.96 & [30] \\
\hline${ }_{20}^{40} \mathrm{Ca}+{ }_{20}^{48} \mathrm{Ca}$ & 400 & 52.0 & - & 53.97 & 9.92 & 54.01 & 9.91 & 52.59 & 52.65 & {$[30]$} \\
\hline${ }_{20}^{40} \mathrm{Ca}+{ }_{22}^{48} \mathrm{Ti}$ & 440 & 58.17 & 9.40 & 59.68 & 9.82 & 59.64 & 9.84 & 57.58 & 57.92 & {$[22]$} \\
\hline${ }_{17}^{35} \mathrm{Cl}+{ }_{26}^{54} \mathrm{Fe}$ & 442 & 61.75 & 9.51 & 60.00 & 9.83 & 59.95 & 9.84 & 57.76 & 58.20 & [29] \\
\hline${ }_{17}^{35} \mathrm{Cl}+{ }_{26}^{56} \mathrm{Fe}$ & 442 & 60.2 & 9.78 & 59.62 & 9.90 & 59.61 & 9.90 & 57.63 & 57.94 & [29] \\
\hline${ }_{16}^{32} S+{ }_{28}^{58} N i$ & 448 & 61.9 & 9.62 & 60.96 & 9.78 & 60.83 & 9.82 & 58.45 & 59.02 & [29] \\
\hline${ }_{17}^{35} \mathrm{Cl}+{ }_{28}^{58} \mathrm{Ni}$ & 476 & 63.00 & 10.10 & 64.13 & 9.92 & 64.05 & 9.91 & 61.67 & 62.13 & [29] \\
\hline${ }_{17}^{35} \mathrm{Cl}+{ }_{28}^{60} \mathrm{Ni}$ & 476 & 62.29 & 10.22 & 63.74 & 9.99 & 63.71 & 9.97 & 61.54 & 61.87 & [29] \\
\hline${ }_{17}^{35} \mathrm{Cl}+{ }_{28}^{62} \mathrm{Ni}$ & 476 & 61.9 & 10.29 & 63.37 & 10.05 & 63.39 & 10.03 & 61.40 & 61.61 & {$[29]$} \\
\hline${ }_{17}^{35} \mathrm{Cl}+{ }_{28}^{64} \mathrm{Ni}$ & 476 & 60.71 & 10.51 & 63.03 & 10.07 & 63.07 & 10.09 & 61.27 & 61.36 & [29] \\
\hline${ }_{8}^{16} \mathrm{O}+{ }_{60}^{148} \mathrm{Nd}$ & 480 & 59.2 & 10.90 & 59.86 & 10.78 & 59.85 & 10.83 & 58.43 & 58.86 & [29] \\
\hline${ }_{8}^{16} \mathrm{O}+{ }_{60}^{150} \mathrm{Nd}$ & 480 & 59.2 & 10.90 & 59.70 & 10.76 & 59.69 & 10.87 & 58.35 & 58.73 & [29] \\
\hline${ }_{8}^{18} \mathrm{O}+{ }_{60}^{148} \mathrm{Nd}$ & 480 & 59.6 & 10.82 & 59.04 & 10.92 & 59.20 & 10.97 & 58.27 & 58.32 & [29] \\
\hline${ }_{22}^{46} T i+{ }_{22}^{46} T i$ & 484 & 66.30 & - & 65.01 & 9.94 & 64.98 & 9.93 & 62.70 & 63.03 & {$[30]$} \\
\hline${ }_{6}^{12} \mathrm{C}+{ }_{82}^{204} \mathrm{~Pb}$ & 492 & 57.55 & - & 59.01 & 11.19 & 59.03 & 11.32 & 57.64 & 58.38 & [30] \\
\hline
\end{tabular}




\begin{tabular}{|c|c|c|c|c|c|c|c|c|c|c|}
\hline${ }_{8}^{16} \mathrm{O}+{ }_{62}^{144} \mathrm{Sm}$ & 496 & 59.9 & - & 62.29 & 10.66 & 62.23 & 10.74 & 60.41 & 61.08 & {$[2]$} \\
\hline${ }_{8}^{16} \mathrm{O}+{ }_{62}^{148} \mathrm{Sm}$ & 496 & 60.53 & 11.02 & 61.95 & 10.73 & 61.91 & 10.81 & 60.25 & 60.82 & [29] \\
\hline${ }_{8}^{16} \mathrm{O}+{ }_{62}^{150} \mathrm{Sm}$ & 496 & 60.2 & 11.08 & 61.78 & 10.76 & 61.75 & 10.84 & 60.17 & 60.69 & [29] \\
\hline${ }_{8}^{16} \mathrm{O}+{ }_{62}^{152} \mathrm{Sm}$ & 496 & 60.2 & 11.09 & 61.62 & 10.80 & 61.59 & 10.87 & 60.10 & 60.56 & [29] \\
\hline${ }_{8}^{16} \mathrm{O}+{ }_{62}^{154} \mathrm{Sm}$ & 496 & 59.65 & 11.20 & 61.46 & 10.85 & 61.43 & 10.91 & 60.02 & 60.43 & [29] \\
\hline${ }_{18}^{40} A r+{ }_{28}^{58} N i$ & 504 & 66.32 & 10.16 & 66.76 & 10.07 & 66.78 & 10.08 & 64.68 & 64.85 & [29] \\
\hline${ }_{18}^{40} A r+{ }_{28}^{60} N i$ & 504 & 65.5 & - & 66.36 & 10.14 & 66.43 & 10.14 & 64.55 & 64.58 & {$[2]$} \\
\hline${ }_{18}^{40} A r+{ }_{28}^{62} N i$ & 504 & 65.1 & - & 65.98 & 10.20 & 66.09 & 10.20 & 64.41 & 64.32 & {$[2]$} \\
\hline${ }_{18}^{40} A r+{ }_{28}^{64} N i$ & 504 & 63.9 & - & 65.63 & 10.27 & 65.77 & 10.26 & 64.27 & 64.06 & {$[2]$} \\
\hline${ }_{17}^{37} \mathrm{Cl}+{ }_{30}^{68} \mathrm{Zn}$ & 510 & 65.9 & 8.5 & 66.52 & 10.23 & 66.63 & 10.24 & 64.95 & 64.86 & [28] \\
\hline${ }_{20}^{40} \mathrm{Ca}+{ }_{28}^{58} \mathrm{Ni}$ & 560 & 73.36 & 10.20 & 74.72 & 9.98 & 74.52 & 10.01 & 71.49 & 72.06 & {$[2]$} \\
\hline${ }_{20}^{40} \mathrm{Ca}+{ }_{28}^{62} \mathrm{Ni}$ & 560 & 72.30 & 10.35 & 73.84 & 10.11 & 73.75 & 10.13 & 71.19 & 71.46 & [29] \\
\hline${ }_{14}^{28} S i+{ }_{40}^{92} Z r$ & 560 & 70.93 & - & 72.36 & 10.33 & 72.27 & 10.37 & 70.05 & 70.31 & {$[23]$} \\
\hline${ }_{8}^{16} O+{ }_{74}^{186} W$ & 592 & 68.87 & 10.60 & 71.12 & 11.18 & 71.02 & 11.25 & 69.51 & 69.93 & {$[24]$} \\
\hline${ }_{22}^{48} T i+{ }_{28}^{58} N i$ & 616 & 78.80 & - & 80.28 & 10.23 & 80.24 & 10.24 & 77.51 & 77.68 & {$[25]$} \\
\hline${ }_{16}^{36} S+{ }_{40}^{90} Z r$ & 640 & 79.00 & 10.80 & 80.76 & 10.58 & 80.85 & 10.59 & 78.87 & 78.66 & {$[26]$} \\
\hline${ }_{8}^{16} \mathrm{O}+{ }_{82}^{208} \mathrm{~Pb}$ & 656 & 75.41 & 11.74 & 77.32 & 11.38 & 77.15 & 11.47 & 75.63 & 76.05 & [29] \\
\hline${ }_{17}^{35} \mathrm{Cl}+{ }_{40}^{90} \mathrm{Zr}$ & 680 & 84.87 & 10.74 & 86.50 & 10.51 & 86.41 & 10.50 & 83.68 & 83.81 & {$[2]$} \\
\hline${ }_{17}^{35} \mathrm{Cl}+{ }_{40}^{92} \mathrm{Zr}$ & 680 & 82.94 & - & 86.16 & 10.53 & 86.09 & 10.55 & 83.54 & 83.56 & {$[23]$} \\
\hline${ }_{9}^{19} F+{ }_{79}^{197} A u$ & 711 & 81.61 & - & 83.55 & 11.46 & 83.50 & 11.46 & 81.98 & 82.13 & {$[30]$} \\
\hline${ }_{8}^{16} O+{ }_{92}^{238} U$ & 736 & 83.2 & - & 84.70 & 11.65 & 84.50 & 11.75 & 83.00 & 83.37 & [18] \\
\hline${ }_{17}^{35} \mathrm{Cl}+{ }_{46}^{106} \mathrm{Pd}$ & 782 & 94.30 & - & 97.26 & 10.75 & 97.13 & 10.74 & 94.21 & 94.22 & {$[30]$} \\
\hline${ }_{28}^{58} N i+{ }_{28}^{58} N i$ & 784 & 95.80 & - & 100.60 & 10.36 & 100.36 & 10.39 & 96.33 & 96.72 & {$[27]$} \\
\hline${ }_{28}^{58} N i+{ }_{28}^{60} N i$ & 784 & 96.0 & 7.5 & 100.01 & 10.47 & 99.85 & 10.45 & 96.13 & 96.34 & {$[30]$} \\
\hline${ }_{28}^{64} N i+{ }_{28}^{64} N i$ & 784 & 93.5 & - & 97.32 & 10.79 & 97.43 & 10.74 & 95.15 & 94.50 & {$[18]$} \\
\hline${ }_{16}^{32} S+{ }_{50}^{116} S n$ & 800 & 97.36 & - & 99.16 & 10.78 & 98.93 & 10.79 & 95.81 & 95.98 & [30] \\
\hline${ }_{20}^{40} \mathrm{Ca}+{ }_{40}^{90} \mathrm{Zr}$ & 800 & 96.88 & 10.00 & 100.86 & 10.57 & 100.63 & 10.59 & 97.08 & 97.28 & [30] \\
\hline${ }_{18}^{40} \mathrm{Ar}+{ }_{46}^{110} \mathrm{Pd}$ & 828 & 101.8 & 10.89 & 100.68 & 11.04 & 100.73 & 10.98 & 98.58 & 97.95 & {$[29]$} \\
\hline${ }_{18}^{40} A r+{ }_{47}^{109} A g$ & 846 & 105.1 & 10.77 & 103.15 & 10.96 & 103.18 & 10.94 & 100.68 & 100.21 & {$[2 !$} \\
\hline
\end{tabular}




\begin{tabular}{|c|c|c|c|c|c|c|c|c|c|c|}
\hline${ }_{17}^{35} \mathrm{Cl}+{ }_{50}^{112} \mathrm{Sn}$ & 850 & 106.0 & 10.72 & 105.10 & 10.82 & 104.91 & 10.80 & 101.44 & 101.62 & [29] \\
\hline${ }_{17}^{35} \mathrm{Cl}+{ }_{50}^{116} \mathrm{Sn}$ & 850 & 105.2 & 10.81 & 104.40 & 10.91 & 104.25 & 10.87 & 101.14 & 101.11 & [29] \\
\hline${ }_{17}^{35} \mathrm{Cl}+{ }_{50}^{120} \mathrm{Sn}$ & 850 & 106.4 & 10.66 & 103.74 & 10.93 & 103.61 & 10.95 & 100.85 & 100.62 & [29] \\
\hline${ }_{17}^{35} C l+{ }_{50}^{124} S n$ & 850 & 105.3 & 10.79 & 103.12 & 11.01 & 102.99 & 11.02 & 100.56 & 100.15 & [29] \\
\hline${ }_{18}^{40} A r+{ }_{50}^{112} S n$ & 900 & 109.1 & - & 109.55 & 10.97 & 109.52 & 10.95 & 106.47 & 106.20 & {$[2]$} \\
\hline${ }_{18}^{40} A r+{ }_{50}^{116} S n$ & 900 & 108.3 & - & 108.82 & 11.06 & 108.84 & 11.03 & 106.17 & 105.68 & {$[2]$} \\
\hline${ }_{18}^{40} A r+{ }_{50}^{122} S n$ & 900 & 107.2 & - & 107.83 & 11.18 & 107.86 & 11.14 & 105.72 & 104.92 & {$[2]$} \\
\hline${ }_{18}^{40} A r+{ }_{51}^{121} S b$ & 918 & 111.7 & 11.00 & 110.25 & 11.15 & 110.27 & 11.11 & 107.78 & 107.15 & {$[29]$} \\
\hline${ }_{17}^{35} \mathrm{Cl}+{ }_{59}^{141} \mathrm{Pr}$ & 1003 & 121.3 & 11.06 & 119.70 & 11.22 & 119.49 & 11.19 & 116.19 & 115.96 & [29] \\
\hline${ }_{36}^{86} K r+{ }_{28}^{58} N i$ & 1008 & 120.8 & - & 122.77 & 10.95 & 122.65 & 10.93 & 119.02 & 118.48 & {$[2]$} \\
\hline${ }_{36}^{86} K r+{ }_{28}^{62} N i$ & 1008 & 119.3 & - & 121.43 & 11.11 & 121.48 & 11.04 & 118.55 & 117.59 & {$[2]$} \\
\hline${ }_{36}^{86} K r+{ }_{28}^{64} N i$ & 1008 & 118.3 & - & 120.82 & 11.16 & 120.92 & 11.10 & 118.31 & 117.16 & {$[2]$} \\
\hline${ }_{32}^{74} G e+{ }_{32}^{74} G e$ & 1024 & 120.8 & - & 123.06 & 11.13 & 123.16 & 11.07 & 120.25 & 119.20 & {$[2]$} \\
\hline${ }_{18}^{40} \mathrm{Ar}+{ }_{62}^{144} \mathrm{Sm}$ & 1116 & 129.97 & 11.53 & 130.96 & 11.37 & 130.92 & 11.35 & 127.57 & 127.01 & {$[29]$} \\
\hline${ }_{18}^{40} A r+{ }_{62}^{148} S m$ & 1116 & 129.56 & 11.57 & 130.28 & 11.44 & 130.25 & 11.41 & 127.26 & 126.50 & {$[29]$} \\
\hline${ }_{18}^{40} \mathrm{Ar}+{ }_{62}^{154} \mathrm{Sm}$ & 1116 & 125.19 & 12.02 & 129.3 & 11.55 & 129.28 & 11.50 & 126.80 & 125.75 & [29] \\
\hline${ }_{36}^{86} K r+{ }_{32}^{70} G e$ & 1152 & 136.5 & - & 137.16 & 11.2 & 137.18 & 11.16 & 133.54 & 132.52 & [18] \\
\hline${ }_{18}^{40} \mathrm{Ar}+{ }_{67}^{165} \mathrm{Ho}$ & 1206 & 141.38 & 11.48 & 138.4 & 11.65 & 138.35 & 11.60 & 135.52 & 134.49 & [29] \\
\hline${ }_{18}^{40} \mathrm{Ar}+{ }_{69}^{169} \mathrm{Tm}$ & 1242 & 139 & 10.5 & 142.05 & 11.68 & 142.01 & 11.64 & 139.01 & 138.01 & {$[2]$} \\
\hline${ }_{18}^{40} A r+{ }_{70}^{174} Y b$ & 1260 & 139 & 9.50 & 143.39 & 11.76 & 143.35 & 11.70 & 140.52 & 139.39 & {$[2]$} \\
\hline${ }_{18}^{40} A r+{ }_{71}^{175} L u$ & 1278 & 140 & 10.0 & 145.37 & 11.77 & 145.33 & 11.70 & 142.33 & 141.26 & {$[2]$} \\
\hline${ }_{18}^{40} A r+{ }_{72}^{176} \mathrm{Hf}$ & 1296 & 143 & 9.50 & 147.35 & 11.75 & 147.30 & 11.70 & 144.13 & 143.12 & {$[2]$} \\
\hline${ }_{18}^{40} \mathrm{Ar}+{ }_{72}^{177} \mathrm{Hf}$ & 1296 & 145 & 10.5 & 147.19 & 11.76 & 147.14 & 11.72 & 144.05 & 143.00 & {$[2]$} \\
\hline${ }_{18}^{40} \mathrm{Ar}+{ }_{72}^{178} \mathrm{Hf}$ & 1296 & 143 & 10.0 & 147.03 & 11.78 & 146.98 & 11.73 & 143.98 & 142.87 & {$[2]$} \\
\hline${ }_{18}^{40} \mathrm{Ar}+{ }_{72}^{179} \mathrm{Hf}$ & 1296 & 144 & 10.2 & 146.87 & 11.79 & 146.82 & 11.74 & 143.90 & 142.75 & \\
\hline${ }_{18}^{40} \mathrm{Ar}+{ }_{72}^{180} \mathrm{Hf}$ & 1296 & 143 & 10.1 & 146.71 & 11.81 & 146.67 & 11.76 & 143.82 & 142.63 & \\
\hline${ }_{16}^{32} S+{ }_{82}^{208} \mathrm{~Pb}$ & 1312 & 153 & - & 148.5 & 11.78 & 148.04 & 11.79 & 144.43 & 144.00 & {$[1]$} \\
\hline${ }_{35}^{81} \mathrm{Br}+{ }_{40}^{90} \mathrm{Zr}$ & 1400 & 156 & - & 163.29 & 11.42 & 163.32 & 11.36 & 158.59 & 157.39 & [0 \\
\hline${ }_{35}^{81} \mathrm{Br}+{ }_{40}^{94} \mathrm{Zr}$ & 1400 & 154 & - & 162.09 & 11.51 & 162.19 & 11.45 & 158.09 & 156.53 & {$[\circ$} \\
\hline
\end{tabular}




\begin{tabular}{|c|c|c|c|c|c|c|c|c|c|c|}
\hline${ }_{17}^{37} \mathrm{Cl}+{ }_{83}^{209} \mathrm{Bi}$ & 1411 & 156.2 & - & 157.15 & 11.96 & 157.03 & 11.95 & 153.87 & 152.79 & {$[18$} \\
\hline${ }_{18}^{40} A r+{ }_{79}^{197} A u$ & 1422 & 164.18 & 11.65 & 158.8 & 11.97 & 158.75 & 11.90 & 155.56 & 154.33 & {$[1]$} \\
\hline${ }_{35}^{81} \mathrm{Br}+{ }_{42}^{96} \mathrm{Mo}$ & 1470 & 174 & - & 170.05 & 11.52 & 170.11 & 11.45 & 165.31 & 163.93 & {$[0]$} \\
\hline${ }_{18}^{40} A r+{ }_{82}^{206} \mathrm{~Pb}$ & 1476 & 158.5 & - & 163.7 & 12.06 & 163.60 & 11.99 & 160.39 & 159.07 & [1] \\
\hline${ }_{35}^{81} B r+{ }_{44}^{104} R u$ & 1540 & 174 & - & 176.18 & 11.61 & 176.29 & 11.57 & 171.73 & 170.00 & {$[2]$} \\
\hline${ }_{40}^{90} Z r+{ }_{40}^{90} Z r$ & 1600 & 182 & - & 184.8 & 11.48 & 184.77 & 11.46 & 178.69 & 177.58 & \\
\hline${ }_{40}^{90} Z r+{ }_{40}^{96} Z r$ & 1600 & 184.5 & - & 182.8 & 11.61 & 182.89 & 11.58 & 177.86 & 176.17 & \\
\hline${ }_{40}^{96} Z r+{ }_{40}^{96} Z r$ & 1600 & 181.4 & - & 180.9 & 11.75 & 181.06 & 11.71 & 177.05 & 174.78 & \\
\hline${ }_{20}^{48} \mathrm{Ca}+{ }_{82}^{208} \mathrm{~Pb}$ & 1640 & 176 & - & 178.3 & 12.27 & 178.44 & 12.20 & 175.76 & 173.51 & \\
\hline${ }_{18}^{40} A r+{ }_{92}^{238} U$ & 1656 & 185 & - & 179.3 & 12.30 & 179.23 & 12.26 & 176.10 & 174.41 & \\
\hline${ }_{42}^{100} \mathrm{Mo}+{ }_{40}^{90} \mathrm{Zr}$ & 1680 & 195.9 & - & 191.16 & 11.63 & 191.23 & 11.62 & 185.73 & 184.04 & \\
\hline${ }_{42}^{100} \mathrm{Mo}+{ }_{42}^{100} \mathrm{Mo}$ & 1764 & 207.2 & - & 197.77 & 11.85 & 197.96 & 11.79 & 193.07 & 190.76 & \\
\hline${ }_{22}^{50} \mathrm{Ti}+{ }_{82}^{208} \mathrm{~Pb}$ & 1804 & 191 & - & 196.1 & 12.25 & 196.16 & 12.18 & 192.19 & 190.12 & \\
\hline${ }_{24}^{54} \mathrm{Cr}+{ }_{76}^{196} \mathrm{Os}$ & 1824 & 203.5 & - & 198.8 & 12.20 & 198.83 & 12.15 & 194.83 & 192.54 & \\
\hline${ }_{22}^{50} T i+{ }_{83}^{209} B i$ & 1826 & 190 & - & 198.4 & 12.21 & 198.47 & 12.18 & 194.30 & 192.30 & \\
\hline${ }_{36}^{86} K r+{ }_{51}^{123} S b$ & 1836 & 209.4 & - & & & & & & 196.91 & \\
\hline${ }_{20}^{48} \mathrm{Ca}+{ }_{96}^{248} \mathrm{Cm}$ & 1920 & 210.0 & - & & & & 12.52 & & 197.59 & \\
\hline${ }_{24}^{52} \mathrm{Cr}+{ }_{82}^{208} \mathrm{~Pb}$ & 1968 & 213.4 & - & 214.0 & 12.17 & 213.83 & 12.17 & 208.48 & 206.62 & \\
\hline${ }_{24}^{54} \mathrm{Cr}+{ }_{83}^{209} \mathrm{Bi}$ & 1992 & 212 & - & 215.3 & 12.25 & 215.36 & 12.23 & 210.37 & 208.22 & \\
\hline${ }_{50}^{124} S n+{ }_{40}^{94} Z r$ & 2000 & 219.5 & - & 219.9 & 12.06 & 220.16 & 12.00 & 214.88 & 212.03 & \\
\hline${ }_{26}^{58} \mathrm{Fe}+{ }_{82}^{208} \mathrm{~Pb}$ & 2132 & 235.6 & - & 229.4 & 12.26 & 229.40 & 12.27 & 223.80 & 221.46 & \\
\hline${ }_{26}^{58} \mathrm{Fe}+{ }_{83}^{209} \mathrm{Bi}$ & 2158 & 236.5 & - & 232.1 & 12.27 & 232.11 & 12.28 & 226.26 & 223.99 & \\
\hline${ }_{32}^{76} G e+{ }_{68}^{170} \mathrm{Er}$ & 2176 & 237 & - & 234.5 & 12.29 & 234.82 & 12.23 & 229.47 & 226.38 & \\
\hline${ }_{29}^{63} C u+{ }_{79}^{197} A u$ & 2291 & 233 & - & 247.5 & 12.07 & 247.31 & 12.21 & 240.01 & 237.94 & \\
\hline${ }_{28}^{64} \mathrm{Ni}+{ }_{82}^{208} \mathrm{~Pb}$ & 2296 & 267.2 & - & 244.7 & 12.33 & 244.84 & 12.37 & 238.95 & 236.14 & \\
\hline${ }_{36}^{86} K r+{ }_{64}^{160} G d$ & 2304 & $\geq 255$ & - & 247.4 & 12.28 & 247.69 & 12.27 & 241.84 & 238.46 & \\
\hline${ }_{26}^{58} F e+{ }_{92}^{238} U$ & 2392 & 280 & - & 253.5 & 12.30 & 252.11 & 12.51 & 246.12 & 243.36 & \\
\hline${ }_{36}^{84} K r+{ }_{67}^{165} \mathrm{Ho}$ & 2412 & 278 & - & 259.1 & 12.18 & 259.34 & 12.25 & 252.25 & 249.23 & \\
\hline${ }_{54}^{136} \mathrm{Xe}+{ }_{46}^{110} \mathrm{Pd}$ & 2484 & $\geq 280$ & - & 265.4 & 12.30 & 265.67 & 12.31 & 259.10 & 255.30 & \\
\hline
\end{tabular}




\begin{tabular}{|l|l|ll|ll|ll|l|l|l|}
${ }_{36}^{84} K r+{ }_{83}^{209} B i$ & 2988 & 365 & - & 311.7 & 12.19 & 311.10 & 12.62 & 302.25 & 298.46 & {$[1]$} \\
\hline
\end{tabular}

There is an overall agreement for the fusion barrier heights obtained from the GLDM for most of the reactions. For the medium systems there is a tendency for our theoretical results to be a little higher than the empirical data, the KNS potential [37] giving still higher values as well as the frozen-density proximity. For the heaviest systems the theoretical data are systematically lower than the experimental ones. The heavier the system is, the smaller the distance between the position of the top of the barrier and the contact point [1]. For $Z_{1} Z_{2} \geq 2100$ this distance is smaller than $1 \mathrm{fm}$. A system put at the top of the barrier without excitation energy does not descend automatically to the ground state due to the friction forces correlated to the high value of the proximity energy. A so-called 'extra-push' energy is needed to overcome the barrier. The values obtained by the formula (1) [18] giving the fusion barrier height are close to the experimental data and the theoretical values given by the GLDM.

In Table 2, for the fusion barrier height, the root-mean-square deviation between the experimental data and the values given by the GLDM and the different formulas are shown. The GLDM is more precise that the different formulas. The rms deviations are large but the experimental error bars are also relatively large, particularly for the heaviest systems. The accuracy of the formula (4) may be improved in choosing for the root-mean-square radii $<r_{i}^{2}>^{1 / 2}$ the experimental data.

Table 2: For the fusion barrier height (in $\mathrm{MeV}$ ), rootmean-square deviation between the experimental data and, successively, the values given by the GLDM, by the formulas (1), (3), (6) and (8).

\begin{tabular}{|c|c|c|c|c|c|}
\hline$Z_{1} Z_{2}$ range & GLDM & {$[18]$} & {$[32,33]$} & {$[34]$} & {$[35]$} \\
\hline $20<Z_{1} Z_{2}<2000$ & 2.34 & 2.38 & 2.83 & 3.29 & 3.35 \\
$Z_{1} Z_{2}<1000$ & 1.28 & 1.28 & 1.40 & 1.40 & 1.51 \\
$1000<Z_{1} Z_{2}<2000$ & 4.28 & 4.37 & 5.3 & 6.35 & 6.42 \\
\hline
\end{tabular}

The comparison of the position of the fusion barrier predicted empirically and theoretically is difficult since several different approaches have been used: interaction barrier, Coulomb peak, linear interpolation,... Our predictions for the barrier position are very similar to the ones in $[29,37]$. In Table 3 , the rootmean-square deviation between the experimental data and the values given by the model and the formulas are given. 
Table 3: For the fusion barrier radius (in fm), root-meansquare deviation between the experimental data and, successively, the values given by the GLDM and by the formulas (2), (4), (7) and (9).

\begin{tabular}{|c|c|c|c|c|}
\hline GLDM & {$[18]$} & {$[32,33]$} & {$[34]$} & {$[35]$} \\
\hline 0.68 & 0.67 & 0.78 & 0.87 & 0.65 \\
\hline
\end{tabular}

\section{Fission barrier}

The fission barriers have been investigated within this GLDM and in the often called 'cold fission valley' roughly described by these quasimolecular shapes (elliptic lemniscatoids [1]) leading rapidly to touching spherical fragments which separate after in keeping the spherical shapes. The microscopic corrections (shell and pairing effects) are included at the beginning of the fission process to determine the ground state. The path allowing the ellipsoidal deformations of the fragments and taking into account the associated microscopic corrections has been also studied.

As an example, the barriers which minimise the barrier height in the exit channel are displayed for 3 fissioning nuclei in Fig. 2. Their heights are close to the experimental fission barrier data $[38,39]$. The saddle-point corresponds to two separated spherical fragments.

It has been shown that the dependence on the asymmetry $\left(A_{1}-A_{2}\right) /\left(A_{1}+A_{2}\right)$

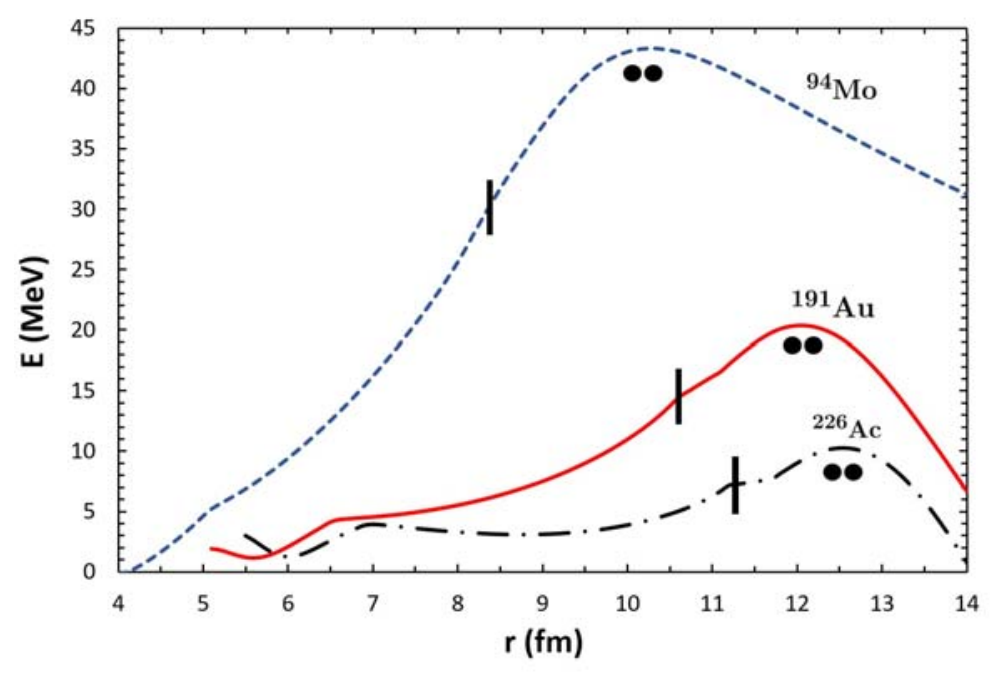

Fig. 2. Fission barriers of the ${ }_{42}^{94} M o,{ }_{79}^{191} A u$ and ${ }_{89}^{226} A c$ nuclei versus the distance between the mass centres. The vertical dash indicates the contact point between the two spherical nuclei. The fragment deformation is not taken into account. 
of the fission of the ${ }^{70,76} \mathrm{Se},{ }^{75} \mathrm{Br},{ }^{90,94,98} \mathrm{Mo},{ }^{110-112} \mathrm{In},{ }^{149} \mathrm{~Tb}$ and ${ }^{194} \mathrm{Hg}$ nuclei is correctly reproduced within the GLDM $[40,41]$. For these nuclei, our calculated asymmetric fission barrier heights lie between the predictions of the Yukawa plus Exponential Finite-Range Liquid-Drop Model [37] and the Liquid Drop Model [42].

The potential energy surface depending on the asymmetry of the decay and the distance between the mass centres is displayed in Fig. 3 and 4 for the ${ }_{78}^{192} \mathrm{Pt}$ and ${ }_{47}^{99} \mathrm{Ag}$. The deformation of the fragments has not been taken into account. In one case a smooth saddle appears favoring the symmetric exit channel while in the other case a plateau appears and a rapid lowering for the very asymmetric decay channel. As an example the dependence of the potential energy

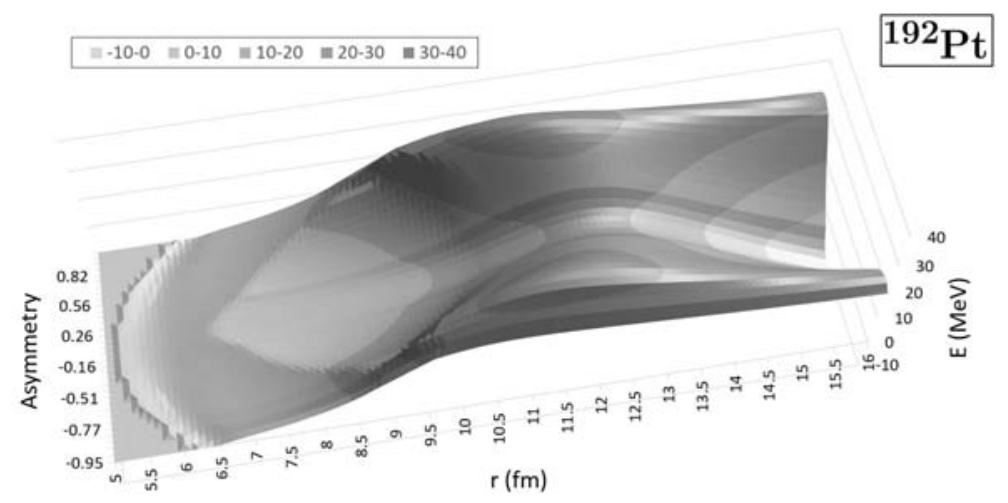

Fig. 3. Potential energy surface as a function of the asymmetry $\alpha=\left(A_{1}-A_{2}\right) /\left(A_{1}+A_{2}\right)$ and the distance $\mathrm{r}$ between the mass centres for ${ }_{78} \mathrm{Pt}$.

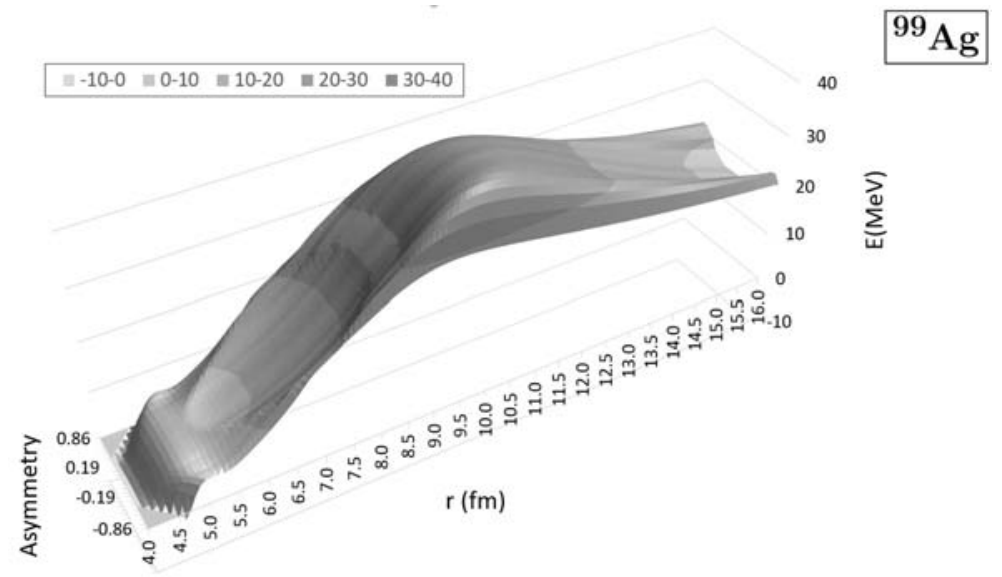

Fig. 4. Potential energy surface as a function of the asymmetry $\alpha=\left(A_{1}-A_{2}\right) /\left(A_{1}+A_{2}\right)$ and the distance $\mathrm{r}$ between the mass centres for ${ }_{47}^{99} \mathrm{Ag}$.

on the shape sequence and on the introduction of the microscopic corrections is displayed in Fig. 5 for the symmetric fission path of the ${ }^{228} R a$ nucleus. 


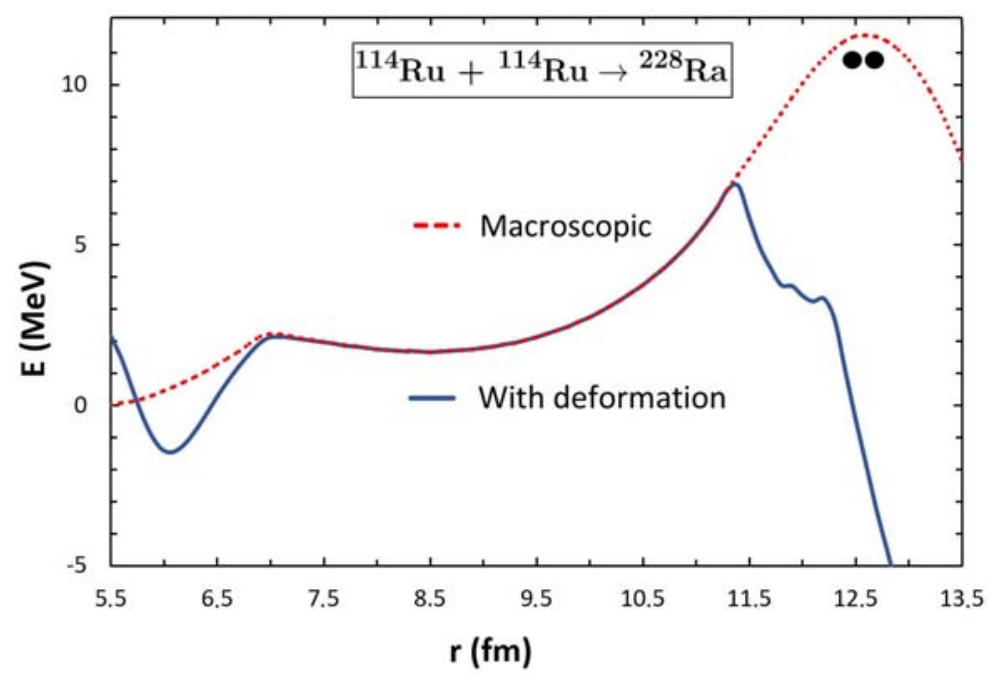

Fig. 5. Symmetric fission barrier of a ${ }^{228} R a$ nucleus. The dashed curve gives the macroscopic energy within the two-sphere approximation while the solid line includes the microscopic corrections around the sphere and the ellipsoidal deformations and the shell and pairing energies of the fragments. $\mathrm{r}$ is the distance between the mass centres.

More generally, the height of the lowest theoretical potential barrier encountered in the fission channel is compared with the experimental data $[1,4,10,38,39,43]$ in Table 4 assuming or not the deformation and microscopic effects of the fragments. The error bar of these experimental data is often of several MeV. For all the nuclei the experimental values lie between the values given by the GLDM and neglecting the microscopic effects in the fragments and the values determined in including the deformation, shell and pairing effects of the fragments. The table is limited to nuclei of charge $Z \leq 89$ since the doublehumped fission barriers of actinides have been studied in a previous work [5]. The indicated prefered exit channel for each fissioning nucleus is only a rough indication since there are often almost degeneracy in energy between several exit channels. The nuclear fission-fragment distributions are smooth [44]. To reproduce the fission barrier heights of the $R a$ and $A c$ nuclei and light isotopes of $\mathrm{Hg}$ it is necessary to introduce the microscopic effects and deformation of the fragments (See also [45]).

Long time ago the fission valley were firstly investigated assuming that the fission process is only governed by the balance between the repulsive Coulomb force and the attractive surface tension force [46,47]. Later on, the fusion studies have shown that for two-body shapes and one-body strongly distorted and creviced shapes it is necessary to add at the surface energy an additional term, the nuclear proximity energy [48], to reproduce the experimental fusion barrier heights. This proximity energy plays no role and doesn't change the energy for one ellipsoid or shapes with very shallow necks but lowers considerably the energy for compact and creviced shapes and two-body ellipsoidal shapes. So, this proximity energy moves closer together the so-called fission 
valley and fusion valley. Without the proximity energy it is difficult to link the sheet of the potential surface corresponding to one-body shapes and the one which corresponds to two separated fragments. This problem remains in the microscopic approaches [49]. It has also been shown that the moment of inertia and quadrupole moment of the shapes are very close in the two valleys [50]. Recently, an evidence for a new compact symmetric fission mode in light thorium isotopes has been advanced [51]. So, it seems that the description of the fission barrier top by two touching or separated fragments is not incompatible with the experimental fission barrier heights.

It is worth mentioning the tentative to reproduce the fission barrier heights by analytical formulas [52] in the region $50 \leq Z \leq 130$.

Table 4: Comparison of the experimental $[1,4,10,38,39,43]$ fission barrier heights $E_{\exp }$ (in $\mathrm{MeV}$ ) with the heights $E_{t h}$ obtained in the prefered decay channels determined from the GLDM assuming the two-sphere approximation and the heights $E_{t h, d e f}$ obtained when the deformation and microscopic effects of the fragments are taken into account. The theoretical radius $R_{\text {fis }}$ (in $\mathrm{fm}$ ) is also given. The values obtained within the Finite Range Liquid Drop Model [39] are displayed in the last column.

\begin{tabular}{|l|l|c|c|c|c|c|c|}
\hline Isotope & Reaction & $R_{\text {fis }}$ & $E_{t h}$ & $E_{\text {exp }}$ & $E_{\text {th,def }}$ & Ref. & {$[39]$} \\
\hline${ }_{34}^{70} \mathrm{Se}$ & ${ }_{17}^{35} \mathrm{Cl}+{ }_{17}^{35} \mathrm{Cl}$ & 9.45 & 42.36 & 39.40 & 42.07 & {$[39]$} & 37.83 \\
${ }_{34} \mathrm{Se}$ & ${ }_{17}^{38} \mathrm{Cl}+{ }_{17}^{38} \mathrm{Cl}$ & 9.8 & 45.20 & 44.50 & 45.16 & {$[39]$} & 44.08 \\
${ }_{42}^{90} \mathrm{Mo}$ & ${ }_{21}^{45} \mathrm{Sc}+{ }_{21}^{45} \mathrm{Sc}$ & 10.0 & 41.85 & 40.92 & 37.49 & {$[39]$} & 41.07 \\
${ }_{42}^{94} \mathrm{Mo}$ & ${ }_{21}^{47} \mathrm{Sc}+{ }_{21}^{47} \mathrm{Sc}$ & 10.3 & 43.56 & 44.68 & 38.19 & {$[39]$} & 44.39 \\
${ }_{42}^{98} \mathrm{Mo}$ & ${ }_{21}^{49} \mathrm{Sc}+{ }_{21}^{49} \mathrm{Sc}$ & 10.4 & 45.07 & 45.84 & 37.63 & {$[39]$} & 47.06 \\
${ }_{47}^{99} \mathrm{Ag}$ & ${ }_{4}^{7} \mathrm{Be}+{ }_{43}^{92} \mathrm{Tc}$ & 9.43 & 30.84 & 29 & 30.46 & {$[38]$} & - \\
${ }_{48}^{109} \mathrm{Cd}$ & ${ }_{24}^{55} \mathrm{Cr}+{ }_{24}^{54} \mathrm{Cr}$ & 10.6 & 40.46 & 34.0 & 36.9 & {$[4]$} & - \\
${ }_{5}^{129} \mathrm{La}$ & ${ }_{2}^{4} \mathrm{He}+{ }_{55}^{125} \mathrm{Cs}$ & 9.93 & 25.21 & 23 & 21.85 & {$[38]$} & - \\
${ }_{63}^{149} \mathrm{Eu}$ & ${ }_{32}^{76} \mathrm{Ge}+{ }_{31}^{73} \mathrm{Ga}$ & 11.4 & 33.36 & 32.5 & 25.98 & {$[38]$} & - \\
${ }_{65}^{152} \mathrm{~Tb}$ & ${ }_{33}^{77} \mathrm{As}+{ }_{32}^{75} \mathrm{Ge}$ & 11.4 & 31.22 & 29.0 & 25.22 & {$[38]$} & - \\
${ }_{66}^{160} \mathrm{Dy}$ & ${ }_{64}^{156} \mathrm{Gd}+{ }_{2}^{4} \mathrm{He}$ & 10.3 & 30.66 & 27.4 & 26.34 & {$[38]$} & - \\
${ }_{67}^{157} \mathrm{Ho}$ & ${ }_{65}^{153} \mathrm{~Tb}+{ }_{2}^{4} \mathrm{He}$ & 10.3 & 26.22 & 26.5 & 22.25 & {$[38]$} & - \\
${ }_{71}^{173} \mathrm{Lu}$ & ${ }_{65}^{169} \mathrm{Tm}+{ }_{2}^{4} \mathrm{He}$ & 10.5 & 28.67 & 28.0 & 21.28 & {$[38]$} & - \\
${ }_{73}^{175} \mathrm{Ta}$ & ${ }_{37}^{89} \mathrm{Rb}+{ }_{36}^{86} \mathrm{Kr}$ & 11.8 & 28.77 & 25.1 & 21.49 & {$[38]$} & -
\end{tabular}




\begin{tabular}{|c|c|c|c|c|c|c|c|}
\hline${ }_{73}^{179} \mathrm{Ta}$ & ${ }_{37}^{91} R b+{ }_{36}^{88} K r$ & 11.9 & 29.08 & 26.1 & 23.03 & [38] & - \\
\hline${ }_{74}^{178} W$ & ${ }_{37}^{89} R b+{ }_{37}^{89} R b$ & 11.9 & 28.63 & 23.0 & 23.81 & [38] & - \\
\hline${ }_{74}^{179} W$ & ${ }_{36}^{87} K r+{ }_{38}^{92} S r$ & 11.9 & 27.73 & 25.2 & 21.64 & {$[38]$} & - \\
\hline${ }_{74}^{180} W$ & ${ }_{37}^{90} R b+{ }_{37}^{90} R b$ & 11.9 & 28.74 & 28.7 & 25.03 & [38] & - \\
\hline${ }_{74}^{181} W$ & ${ }_{36}^{88} K r+{ }_{38}^{93} S r$ & 12.0 & 27.74 & 23.7 & 21.52 & [38] & - \\
\hline${ }_{74}^{182} W$ & ${ }_{37}^{91} R b+{ }_{37}^{91} R b$ & 12.0 & 28.66 & 26.0 & 23.54 & [38] & - \\
\hline${ }_{74}^{184} W$ & ${ }_{37}^{92} R b+{ }_{37}^{92} R b$ & 12.0 & 28.41 & 24.9 & 24.5 & {$[38]$} & - \\
\hline${ }_{75}^{181} R e$ & ${ }_{37}^{89} R b+{ }_{38}^{92} S r$ & 11.9 & 26.09 & 25.0 & 20.65 & {$[38]$} & - \\
\hline${ }_{75}^{185} R e$ & ${ }_{37}^{91} R b+{ }_{38}^{94} S r$ & 12.0 & 25.86 & 25.0 & 19.36 & {$[38]$} & - \\
\hline${ }_{76}^{186} \mathrm{Os}$ & ${ }_{38}^{93} S r+{ }_{38}^{93} S r$ & 12.0 & 25.45 & 23.4 & 19.87 & [38] & - \\
\hline${ }_{76}^{187} \mathrm{Os}$ & ${ }_{37}^{91} R b+{ }_{39}^{96} Y$ & 12.0 & 24.33 & 22.7 & 19.05 & [38] & - \\
\hline${ }_{76}^{188} O s$ & ${ }_{38}^{94} S r+{ }_{38}^{94} S r$ & 12.1 & 25.12 & 24.2 & 17.67 & [38] & - \\
\hline${ }_{76}^{190} \mathrm{Os}$ & ${ }_{38}^{95} \mathrm{Sr}+{ }_{38}^{95} \mathrm{Sr}$ & 12.1 & 24.64 & 24.8 & 17.91 & [38] & - \\
\hline${ }_{77}^{185} \mathrm{Ir}$ & ${ }_{38}^{91} S r+{ }_{39}^{94} Y$ & 11.95 & 22.85 & 20.4 & 18.45 & {$[38]$} & - \\
\hline${ }_{77}^{187} \mathrm{Ir}$ & ${ }_{38}^{92} S r+{ }_{39}^{95} Y$ & 12.0 & 22.74 & 19.1 & 17.09 & {$[38]$} & - \\
\hline${ }_{77}^{189} \mathrm{Ir}$ & ${ }_{38}^{93} S r+{ }_{39}^{96} Y$ & 12.0 & 22.47 & 22.6 & 17.21 & [38] & - \\
\hline${ }_{77}^{191} \mathrm{Ir}$ & ${ }_{38}^{94} S r+{ }_{39}^{97} Y$ & 12.1 & 22.06 & 23.7 & 15.55 & [38] & - \\
\hline${ }_{78}^{190} \mathrm{Pt}$ & ${ }_{39}^{95} Y+{ }_{39}^{95} Y$ & 12.1 & 21.95 & 19.8 & 16.27 & [38] & 18.80 \\
\hline${ }_{78}^{192} \mathrm{Pt}$ & ${ }_{39}^{96} Y+{ }_{39}^{96} Y$ & 12.1 & 21.66 & 20.7 & 17.56 & {$[38]$} & 20.08 \\
\hline${ }_{78}^{193} \mathrm{Pt}$ & ${ }_{38}^{94} S r+{ }_{40}^{99} Z r$ & 12.1 & 20.82 & 21.9 & 14.32 & {$[38]$} & 20.84 \\
\hline${ }_{78}^{194} \mathrm{Pt}$ & ${ }_{39}^{97} Y+{ }_{39}^{97} Y$ & 12.15 & 22.38 & 22.0 & 15.89 & {$[38]$} & 21.47 \\
\hline${ }_{78}^{196} \mathrm{Pt}$ & ${ }_{39}^{98} Y+{ }_{39}^{98} Y$ & 12.2 & 23.62 & 24.0 & 18.18 & {$[38]$} & 23.01 \\
\hline${ }_{79}^{191} A u$ & ${ }_{39}^{94} Y+{ }_{40}^{97} Z r$ & 12.0 & 19.19 & 18.4 & 14.58 & {$[38]$} & 17.99 \\
\hline${ }_{79}^{195} \mathrm{Au}$ & ${ }_{39}^{96} Y+{ }_{40}^{99} Z r$ & 12.1 & 20.72 & 20.1 & 14.95 & {$[38]$} & 20.54 \\
\hline${ }_{79}^{197} A u$ & ${ }_{39}^{97} Y+{ }_{40}^{100} Z r$ & 12.2 & 22.00 & 22.6 & 15.49 & {$[38]$} & 22.31 \\
\hline${ }_{80}^{182} \mathrm{Hg}$ & ${ }_{40}^{91} Z r+{ }_{40}^{91} Z r$ & 11.8 & 17.24 & 9.0 & 11.3 & [38] & 10.85 \\
\hline${ }_{80}^{186} \mathrm{Hg}$ & ${ }_{40}^{93} Z r+{ }_{40}^{93} Z r$ & 11.9 & 18.27 & 11.5 & 14.7 & {$[38]$} & 12.99 \\
\hline${ }_{80}^{192} \mathrm{Hg}$ & ${ }_{40}^{96} Z r+{ }_{40}^{96} Z r$ & 12.1 & 18.86 & 15.7 & 12.63 & [38] & 16.75 \\
\hline${ }_{80}^{194} \mathrm{Hg}$ & ${ }_{40}^{97} Z r+{ }_{40}^{97} Z r$ & 12.1 & 20.01 & 17.0 & 14.32 & [38] & 18.10 \\
\hline
\end{tabular}




\begin{tabular}{|c|c|c|c|c|c|c|c|}
\hline${ }_{80}^{196} \mathrm{Hg}$ & ${ }_{40}^{98} Z r+{ }_{40}^{98} Z r$ & 12.15 & 21.24 & 19.7 & 14.25 & [38] & 19.65 \\
\hline${ }_{80}^{198} \mathrm{Hg}$ & ${ }_{40}^{99} Z r+{ }_{40}^{99} Z r$ & 12.2 & 22.56 & 20.4 & 15.92 & [38] & 21.60 \\
\hline${ }_{80}^{199} \mathrm{Hg}$ & ${ }_{39}^{97} Y+{ }_{41}^{102} \mathrm{Nb}$ & 10.9 & 17.43 & 22.0 & 16.65 & {$[38]$} & 22.24 \\
\hline${ }_{80}^{200} \mathrm{Hg}$ & ${ }_{40}^{100} Z r+{ }_{40}^{100} Z r$ & 12.2 & 23.95 & 22.9 & 16.40 & [38] & 23.23 \\
\hline${ }_{81}^{200} \mathrm{Tl}$ & ${ }_{40}^{99} Z r+{ }_{41}^{101} \mathrm{Nb}$ & 12.2 & 20.83 & 22.8 & 15.48 & [43] & 21.66 \\
\hline${ }_{81}^{201} \mathrm{Tl}$ & ${ }_{41}^{102} \mathrm{Nb}+{ }_{40}^{99} \mathrm{Zr}$ & 12.2 & 22.35 & 22.3 & 16.9 & [38] & 22.23 \\
\hline${ }_{82}^{198} \mathrm{~Pb}$ & ${ }_{41}^{99} \mathrm{Nb}+{ }_{41}^{99} \mathrm{Nb}$ & 12.2 & 20.05 & 17.0 & 14.58 & [38] & 17.28 \\
\hline${ }_{82}^{204} \mathrm{~Pb}$ & ${ }_{41}^{102} \mathrm{Nb}+{ }_{41}^{102} \mathrm{Nb}$ & 12.3 & 24.43 & 24.0 & 18.3 & {$[38]$} & 21.91 \\
\hline${ }_{82}^{205} \mathrm{~Pb}$ & ${ }_{42}^{105} \mathrm{Mo}+{ }_{40}^{100} \mathrm{Zr}$ & 12.3 & 24.29 & 24.6 & 17.43 & {$[43]$} & 23.08 \\
\hline${ }_{82}^{206} \mathrm{~Pb}$ & ${ }_{41}^{103} \mathrm{Nb}+{ }_{41}^{103} \mathrm{Nb}$ & 12.3 & 26.04 & 26.4 & 19.25 & [38] & 23.94 \\
\hline${ }_{82}^{207} \mathrm{~Pb}$ & ${ }_{42}^{106} \mathrm{Mo}+{ }_{40}^{101} \mathrm{Zr}$ & 12.3 & 25.94 & 26.5 & 18.97 & [38] & 24.76 \\
\hline${ }_{82}^{208} \mathrm{~Pb}$ & ${ }_{41}^{104} \mathrm{Nb}+{ }_{41}^{104} \mathrm{Nb}$ & 12.4 & 27.73 & 28.1 & 21.6 & [38] & 24.95 \\
\hline${ }_{83}^{206} B i$ & ${ }_{42}^{104} \mathrm{Mo}+{ }_{41}^{102} \mathrm{Nb}$ & 12.3 & 21.57 & 22.4 & 16 & [38] & 21.42 \\
\hline${ }_{83}^{207} \mathrm{Bi}$ & ${ }_{42}^{105} \mathrm{Mo}+{ }_{41}^{102} \mathrm{Nb}$ & 12.3 & 23.18 & 21.9 & 17.58 & [38] & 22.28 \\
\hline${ }_{83}^{208} B i$ & ${ }_{42}^{105} \mathrm{Mo}+{ }_{41}^{103} \mathrm{Nb}$ & 12.3 & 23.26 & 23.8 & 17.73 & & 23.23 \\
\hline${ }_{83}^{209} \mathrm{Bi}$ & ${ }_{42}^{106} \mathrm{Mo}+{ }_{41}^{103} \mathrm{Nb}$ & 12.3 & 24.91 & 23.3 & 18.52 & [38] & 23.88 \\
\hline${ }_{83}^{210} B i$ & ${ }_{42}^{106} \mathrm{Mo}+{ }_{41}^{104} \mathrm{Nb}$ & 12.4 & 23.27 & 24.3 & 18.22 & {$[38]$} & 22.74 \\
\hline${ }_{84}^{207} \mathrm{Po}$ & ${ }_{43}^{106} \mathrm{Tc}+{ }_{41}^{101} \mathrm{Nb}$ & 12.3 & 20.67 & 19.3 & 15.51 & {$[43]$} & 20.01 \\
\hline${ }_{84}^{208} \mathrm{Po}$ & ${ }_{42}^{104} M o+{ }_{42}^{104} M o$ & 12.3 & 22.47 & 19.1 & 15.32 & [38] & 20.81 \\
\hline${ }_{84}^{209} \mathrm{Po}$ & ${ }_{43}^{107} \mathrm{Tc}+{ }_{41}^{102} \mathrm{Nb}$ & 12.3 & 22.41 & 21.1 & 17.12 & {$[43]$} & 22.24 \\
\hline${ }_{84}^{210} \mathrm{Po}$ & ${ }_{42}^{105} \mathrm{Mo}+{ }_{42}^{105} \mathrm{Mo}$ & 12.4 & 24.23 & 21.4 & 18.13 & [38] & 22.16 \\
\hline${ }_{84}^{211} \mathrm{Po}$ & ${ }_{43}^{108} \mathrm{Tc}+{ }_{41}^{103} \mathrm{Nb}$ & 12.4 & 22.45 & 19.7 & 17.58 & & 21.33 \\
\hline${ }_{84}^{212} \mathrm{Po}$ & ${ }_{42}^{106} \mathrm{Mo}+{ }_{42}^{106} \mathrm{Mo}$ & 12.4 & 22.57 & 19.5 & 15.92 & & 20.19 \\
\hline${ }_{85}^{212} A t$ & ${ }_{43}^{107} \mathrm{Tc}+{ }_{42}^{105} \mathrm{Mo}$ & 12.4 & 19.82 & 18.6 & 15.71 & & 19.37 \\
\hline${ }_{85}^{213} A t$ & ${ }_{43}^{108} \mathrm{Tc}+{ }_{42}^{105} \mathrm{Mo}$ & 12.4 & 18.80 & 17.0 & 15.37 & {$[38]$} & 18.56 \\
\hline${ }_{86}^{216} R n$ & ${ }_{43}^{108} T c+{ }_{43}^{108} T c$ & 12.4 & 17.69 & 13.1 & 12.9 & & 14.24 \\
\hline${ }_{88}^{208} R a$ & ${ }_{44}^{104} R u+{ }_{44}^{104} R u$ & 12.2 & 11.67 & 5.4 & 6.74 & {$[38]$} & 11.70 \\
\hline${ }_{88}^{225} R a$ & ${ }_{45}^{115} R h+{ }_{43}^{110} T c$ & 12.5 & 10.92 & 9.0 & 7.66 & & 8.40 \\
\hline${ }_{88}^{226} R a$ & ${ }_{44}^{113} R u+{ }_{44}^{113} R u$ & 12.6 & 12.24 & 8.5 & 8.05 & & 8.23 \\
\hline
\end{tabular}




\begin{tabular}{|l|l|c|c|c|c|c|c|}
${ }_{88}^{227} R a$ & ${ }_{43}^{111} T c+{ }_{45}^{116} R h$ & 12.55 & 11.74 & 8.3 & 8.28 & {$[38]$} & 8.02 \\
${ }_{88}^{228} R a$ & ${ }_{44}^{114} R u+{ }_{44}^{114} R u$ & 12.6 & 13.00 & 8.1 & 8.27 & {$[39]$} & 7.59 \\
${ }_{89}^{226} A c$ & ${ }_{44}^{112} R u+{ }_{45}^{114} R h$ & 12.5 & 9.03 & 8.0 & 6.03 & {$[38]$} & 7.71 \\
${ }_{89}^{227} A c$ & ${ }_{44}^{112} R u+{ }_{45}^{115} R h$ & 12.55 & 10.22 & 7.3 & 6.52 & {$[38]$} & 7.53 \\
${ }_{89}^{228} A c$ & ${ }_{44}^{113} R u+{ }_{45}^{115} R h$ & 12.6 & 9.89 & 7.2 & 7.18 & {$[38]$} & 7.24 \\
\hline
\end{tabular}

\section{Summary and conclusion}

The fusion and fission barriers have been investigated within compact and creviced shapes and a Generalized Liquid Drop Model taking into account the proximity forces acting between surfaces in regard, the charge and mass asymmetry and the shell and pairing effects. The heights and positions of these barriers have been compared with the empirical data deduced from the experimental results. There is an overall agreement for the fusion barrier heights for most of the reactions. The fusion barrier heights may also be calculated from a proposed analytic formula. The empirical fission barrier heights lie always between the values given by the GLDM and neglecting the microscopic effects in the fragments and the values determined in including the shell and pairing effects and deformation of the fragments. So, it seems that the description of the fission barrier top by two touching or separated fragments is not incompatible with the experimental fission barrier heights.

Two of us (J.M and M.G) acknowledge the warm hospitality of the Subatech laboratory.

Declaration of competing interest :

The authors declare that they have no known competing financial interests or personal relationships that could have appeared to influence the work reported in this paper.

\section{References}

[1] G. Royer, B. Remaud, Nucl. Phys. A 444 (1985) 477.

[2] G. Royer, J. Phys. G : Nucl. Phys. 12 (1986) 623.

[3] G. Royer, M. Prince, X. Scannell, I. Lele-Cheudjou, A. Samb, Nucl. Phys. A 1000 (2020) 121811.

[4] G. Royer, B. Remaud, J. Phys. G : Nucl. Phys. 10 (1984) 1057. 
[5] G. Royer, M. Jaffré, D. Moreau, Phys. Rev. C 86 (2012) 044326.

[6] G. Royer, R.K. Gupta, V. Yu. Denisov, Nucl. Phys. A 632 (1998) 275.

[7] G. Royer, R. Moustabchir, Nucl. Phys. A 683 (2001) 182.

[8] G. Royer, Nucl. Phys. A 848 (2010) 279.

[9] J.G. Deng, H.F. Zhang, G. Royer, Phys. Rev. C 101 (2020) 034307.

[10] G. Royer, J. Jahan, N. Mokus, Physica Scripta 93 (2018) 094004.

[11] G. Royer, C. Piller, J. Mignen, B. Remaud, Nucl. Phys. A 494 (1989) 267.

[12] Shan-Gui Zhou, Proceedings of the $26^{\text {th }}$ International Nuclear Physics Conference INPC2016 (2017) 373.

[13] V. Baran, M. Colonna, V. Greco, M. Di Toro, Physics Reports 410 (2005) 335.

[14] Yu.Ts. Oganessian, A. Sobiczewski, G.M. Ter-Akopian, Physica Scripta 92 (2017) 023003.

[15] S. Hofmann, J. Phys. G : Nucl. Part. Phys. 42 (2015) 114001.

[16] V. Yu. Denisov, S. Hofmann, Phys. Rev. C 61 (2000) 034606.

[17] D.N. Poenaru, R.A. Gherghescu, Phys. Rev. C 94 (2016) 014309.

[18] R. Moustabchir, G. Royer, Nucl. Phys. A 683 (2001) 266.

[19] D. Shapira et al, Phys. Rev. C 28 (1983) 1148.

[20] C.M. Jachcinski et al, Phys. Rev. C 24 (1981) 2070.

[21] P.R.S. Gomes et al, Nucl. Phys. A 534 (1991) 429.

[22] A.A. Sonzogni et al, Phys. Rev. C 57 (1998) 722.

[23] J.O. Newton et al, Phys. Rev. C 64 (2001) 064608.

[24] J.R. Leigh et al, Phys. Rev. C 52 (1995) 3151.

[25] A.M. Vinodkumar et al, Phys. Rev. C 53 (1996) 803.

[26] A.M. Stefanini et al, Phys. Rev. C 62 (2000) 014601.

[27] H. Timmers et al, Nucl. Phys. A 633 (1998) 421.

[28] A. Chauhan et al, Phys. Rev. C 102 (2020) 064606.

[29] L.C. Vaz, J.M. Alexander, G.R. Satchler, Phys. Rep. 69 (1981) 373.

[30] V. Zanganeh, M. Mirzaei, N. Wang, Communications in Theoretical Physics 64 (2015) 177.

[31] A.M. Stefanini et al, Nucl. Phys. A, 456 (1986) 509.

[32] R. Gharaei, G.L. Zhang, Nucl. Phys. A 990 (2019) 294. 
[33] G.L. Zhang, M. Pan, Int. J. Mod. Phys. E 25 (2016) 1650082.

[34] B. Sahu, C.S. Shastry, J. Phys. G, Nucl. Part. Phys. 25 (1999) 1909.

[35] R. Kumari, R.K. Puri, Nucl. Phys. A 933 (2015) 135.

[36] G. Royer, R. Rousseau, Eur. Phys. J. A 42 (2009) 541.

[37] H.J. Krappe, J.R. Nix, A.J. Sierk, Phys. Rev. C 20 (1979) 992.

[38] M. Dahlinger, D. Vermeulen, K.H. Schmidt, Nucl. Phys. A 376 (1982) 94.

[39] P. Möller, A.J. Sierk, T. Ichikawa, H. Sagawa, At. Data Nucl. Data Tables, 109-110 (2016) 1.

[40] G. Royer, F. Haddad, Phys. Rev. C 51 (1995) 2813.

[41] G. Royer, K. Zbiri, Nucl. Phys. A, 697 (2002) 630.

[42] S. Cohen, F. Plasil, W.J. Swiatecki, Ann. Phys. (N.Y) 82 (1974) 557.

[43] A. Mamdouh, J.M. Pearson, M. Rayet, F. Tondeur, Nucl. Phys. A 644 (1998) 389.

[44] J. Randrup, P. Möller, Phys. Rev. Lett. 106 (2011) 132503.

[45] M. Veselský, et al, Phys. Rev. C 86 (2012) 024308.

[46] S. Cohen, W.J. Swiatecki, Ann.of Phys. 22 (1963) 406.

[47] V.M. Strutinsky, N.Ya. Lyashchenko, Na. Popov, Nucl. Phys. A 46 (1963) 639.

[48] J. Blocki, J. Randrup, W.J. Swiatecki, C.F. Tsang, Ann. Phys., NY 105 (1977) 427.

[49] J.F. Berger, M. Girod, D. Gogny, J. Physique Lett. 42 (1981) 509.

[50] J. Mignen, G. Royer, F. Sebille, Nucl. Phys. A 489 (1988) 461.

[51] A. Chatillon, et al, Phys. Rev. Lett. 124 (2020) 202502.

[52] H.C. Manjunatha, Indian J. Phys. 92 (2018) 507. 